\title{
Evaluación individual de un docente y propuestas de prevención
}

Óscar Cervilla Sáez, Mª Inmaculada Fernández Ávalos, Sara Gómez Regalado. Universidad de Granada

Recepción: 12 de junio de 2015 | Revisión: 20 de julio de 2015 | Aceptación/Publicación: 21 de julio de 2015

Correspondencia: inmafa@correo-ugr.es

Citar: Cervilla, O., Fernandez, M.I. y Gomez, S. (2015). Evaluación individual de un docente y propuesta de prevención. ReiDoCrea, 4, 177-182.

\begin{abstract}
Resumen: El estrés parece estar con más frecuencia en el centro de atención de numerosas investigaciones dada su relevancia en distintos ámbitos entre los que se incluye el laboral. Subyace la importancia de conceptualizar y evaluar aquellas variables relacionadas implicadas en el estrés que pueden influir de algún modo en el ámbito laboral y personal de los trabajadores en la Universidad de Granada. Este estudio presenta una evaluación de los riesgos psicosociales de un docente de Universidad. Método: Estudio evaluativo realizado en la Universidad de Granada con un Catedrático de Universidad, para ello se ha utilizado el instrumento de evaluación ISTAS 21. Resultados: El participante presenta niveles de riesgo alto en dimensiones como exigencias psicológicas y doble presencia. Conclusiones: Destacar la necesidad de una adecuada evaluación y prevención de variables psicosociales que pueden suponer un riesgo laboral.
\end{abstract}

Palabras clave: Evaluación de riesgo psicosocial, Prevención

INDIVIDUAL ASSESSMENT AND PREVENTION PURPOSES

Abstract: Numerous researches are focused on stress, including laboral one. Underlying the importance of conceptualizing and evaluating variables related stress involved in that may have some influence in the workplace and workers at the University of Granada. This study presents an evaluation of psychosocial risks of a University lecturer. Method: Evaluative study conducted at the University of Granada with University Professor, for it has been used an assessment instrument, ISTAS 21. Results: The participant presents high levels of risk in psychological dimensions and dual presence requirements. Conclusions: Emphasize the need for proper assessment and prevention of psychosocial variables that may be a potential risk.

Keywords: Psychosocial Risk Assessment, and Prevention

\section{Introducción}

El concepto de salud laboral, gira en torno a la prevención y la evitación del riesgo, está dando un giro de perspectiva con el objetivo de dar un paso más allá de la mera perspectiva reactiva, hacia una más activa (Moreno-Jiménez y Garrosa, 2013). Situando al propio trabajo como algo más que una actividad con la que se obtiene dinero, de manera que este forme parte de la vida de las personas y una fuente de salud, bienestar y desarrollo.

Existen una serie de factores negativos que pueden influir y repercutir en detrimento de la salud laboral de los trabajadores. Aquí se ubicarían los factores de riesgo laborales y de manera más concreta, se prestarán atención a los factores de riesgo psicosociales. Estos pueden tener efectos generalizados como la respuesta de estrés y alcanzar cierto carácter de gravedad.

El estrés laboral es un fenómeno que viene dándose relativamente desde hace muchos años, sin embargo, solo en las últimas décadas ha recibido la atención que merece. Según Serrano, Moya y Salvador (2009), el estrés laboral queda definido como "un fenómeno que afecta a un alto porcentaje de trabajadores en el mundo industrializado, y que conlleva un alto coste personal, psicosocial y económico". 
El modelo OSI (Indicador de Estrés Ocupacional) indica seis factores generadores de estrés que pueden influir tanto en la salud física como psicológica del trabajador. Dichos estresores proceden de los factores intrínsecos y extrínsecos del trabajo, algunos de ellos son el rol organizacional, el clima, la cultura organizacional, relaciones interpersonales y conflicto de familia trabajo. (Cooper, Sloan y Williams, 1988, citado en López-Araujo, Osca y Peiró, 2007).

En base a lo anterior, para poder detectar los niveles de estrés laboral que presenta el trabajador, se debe recurrir a la evaluación psicosocial, la cual queda definida como "un proceso dirigido a estimar la magnitud de aquellos riesgos que no hayan podido evitarse, obteniendo y facilitando la información necesaria para que puedan tomarse las medidas preventivas que deben adoptarse" (Observatorio Permanente de Riesgos Psicosociales UGT-CEC, citado en Giner, 2012).

En el presente informe se pretende realizar dicho proceso de evaluación y exponer los resultados obtenidos tras administrar el cuestionario ISTAS 21 (CoPsoQ) en un trabajador, con el objetivo de analizar los niveles de estrés y detectar en qué áreas parece estar más presente. Este instrumento está diseñado para la identificación y medición de la exposición a seis grandes grupos de factores de riesgos para la salud de naturaleza psicosocial en el trabajo (ISTAS, 2002). En este caso, se trata de la versión corta de dicho cuestionario, adaptado y traducido para la población española por el grupo de trabajo constituido por el Instituto Sindical de Trabajo, Ambiente y Salud (ISTAS).

En base a los resultados, tendrá lugar una propuesta de intervención, ya que las repercusiones en trabajadores con altos niveles de éstres y/o ansiedad pueden ser mayores tanto en su bienestar físico como mental. Esto queda corroborado con la fuerte evidencia existente entre el burnout y síntomas psicológicos, fisiológicos y sociales entre otros, concretamente, altos niveles de burnout correlacionan de manera general con alcoholismo, enfermedades mentales, problemas cardiovasculares, etc. (Maslach y Jackson, 1981; Zapf, 2002; Ahola, 2007; Schaufeli, 2006; Melamed, Shirom, Toker, Berliner, Y Shapira, 2008; citados en Vladut y Kállay, 2010).

\section{Método}

EI instrumento ISTAS 21 ha sido realizado a un hombre de 48 años, padre de una familia numerosa y profesor catedrático desde hace 5 años en la Facultad de Psicología en la Universidad de Granada (UGR). Su horario laboral se extiende bastante ya que no sólo tiene lugar las horas que imparte clase, sino también sus tutorías y algunos otros trabajos de investigación que desempeña.

Dicho cuestionario fue administrado en su despacho, en el mes de marzo de 2015. En primer lugar, se solicitó su consentimiento para poder realizar el cuestionario ISTAS 21. Más tarde, se procedió a explicarle la finalidad de esta actividad. Una vez dado su permiso, el sujeto cumplimentó el ISTAS 21, con la presencia de los investigadores y se aseguró un entorno libre de ruidos $u$ otras distracciones que pudieran interferirle. Más tarde, cuando terminó de rellenarlo, se corrigió y se puntuaron las diferentes áreas correspondientes a este cuestionario delante del participante para que él mismo pudiera explicar cuál cree que puede ser el motivo de los resultados obtenidos. Finalmente se le agradeció su colaboración. 


\section{Resultados}

En la corrección e interpretación de dicho cuestionario, se obtuvieron unas puntuaciones concretas en cada una de las seis dimensiones correspondientes a este instrumento. En la Tabla 1, se exponen dichos resultados obtenidos.

\begin{tabular}{|c|c|c|c|c|}
\hline \multicolumn{7}{|c|}{ Resultados de la administración del cuestionario ISTAS 21 } \\
\hline APARTADO & $\begin{array}{c}\text { DIMENSIÓN } \\
\text { PSICOSOCIAL }\end{array}$ & $\begin{array}{c}\text { VeRDE: Nivel de exposición } \\
\text { psicosocial más favorable } \\
\text { para la salud. }\end{array}$ & $\begin{array}{c}\text { AMARILLO: Nivel de } \\
\text { exposición psicosocial } \\
\text { intermedio. }\end{array}$ & $\begin{array}{c}\text { ROJO: Nivel de exposición } \\
\text { psicosocial más desfavorable } \\
\text { para la salud. }\end{array}$ \\
\hline $\mathbf{1}$ & Exigencias psicológicas & & & 12 \\
\hline $\mathbf{2}$ & $\begin{array}{c}\text { Trabajo activo y } \\
\text { posibilidades de } \\
\text { desarrollo }\end{array}$ & 35 & & \\
\hline $\mathbf{3}$ & Inseguridad & & 36 & \\
\hline $\mathbf{4}$ & $\begin{array}{c}\text { Apoyo social y } \\
\text { calidad de liderazgo }\end{array}$ & & & \\
\hline $\mathbf{5}$ & Doble presencia & & 12 & \\
\hline $\mathbf{6}$ & Estima & & & \\
\hline
\end{tabular}

En esta tabla se observa que el participante ha obtenido una puntuación de 12 en la dimensión de Exigencias psicológicas; con respecto al área de Trabajo activo y posibilidades de desarrollo el sujeto puntúa 35; asimismo, en la dimensión de Inseguridad obtiene 2 puntos; de la misma manera en el área de Apoyo social y calidad de liderazgo su puntuación es de 36; en cuanto a la dimensión de Doble presencia su puntuación es 9; por último, el participante puntúa 12 en la dimensión de Estima.

Por consiguiente, las escalas en las que el participante ha obtenido puntuaciones correspondientes al nivel de exposición desfavorable para la salud (intervalo rojo) corresponden a: Exigencias Psicológicas y Doble presencia. Mientras que en el nivel de exposición psicosocial intermedio (intervalo amarillo), se han situado: Apoyo social y calidad de liderazgo, Inseguridad y Estima. Finalmente, el área de Trabajo activo y posibilidades de desarrollo se encuentra en el nivel de exposición psicosocial favorable para la salud (intervalo verde).

\section{Discusión}

Con respecto a lo expuesto anteriormente, se procede a resaltas las dimensiones en las que J.L. ha obtenido resultados que muestran nivel de exposición psicosocial desfavorable para la salud: Exigencias psicológicas y Doble presencia.

La primera dimensión que se analiza corresponde a exigencias psicológicas. La explicación subyacente a que esta escala puntúe negativamente, hace referencia al alto nivel de responsabilidad que tiene J.L., ya que gestiona tanto su propio trabajo como el de otras personas. En base a esto, se observa que el participante padece un exceso de carga laboral debido a que su puesto de trabajo conlleva gran rapidez para su resolución. Además, hay que tener en cuenta que sus tareas laborales se extienden en el tiempo, es decir, no sólo trabaja en su despacho, sino también en su casa, por esta acumulación de tareas. Esto demuestra que J.L. no mantiene una distribución regular de sus obligaciones laborales. De hecho, algunas veces tiene dificultades para llevar al día su trabajo, lo que se relaciona con la acumulación de este anteriormente comentada provocando cierto desgaste emocional. Este desgaste emocional también está debido, por ejemplo, a la preocupación que suponen para él que sus alumnos 
obtengan buenos o malos resultados. A pesar de ello, él no percibe que su puesto de trabajo sea estresante, aunque admite que para otras personas externas pueda parecerlo.

Por otra parte, la segunda dimensión destacable por su riesgo, referida a la doble presencia, está relacionada con la conciliación familiar y laboral. Esto es debido a que el sujeto tiene familia numerosa y se añade que su pareja también trabaja como profesora, por lo que su preocupación aumenta. A pesar de que se reparten las tareas equitativamente, algunas veces, las obligaciones laborales interfieren con las necesidades familiares o de encontrarse en casa en ese momento. Además de esto, cuando está en su trabajo, en ocasiones no puede evitar pensar en las tareas del hogar mientras está en la empresa.

Siguiendo en la misma línea de lo anterior, se proponen implantar una serie de medidas de intervención. Dichas medidas serán consideradas en base a dos niveles, individual e institucional, con los que se pretende abarcar de un modo más amplio las dificultades que pueda presentar $\mathrm{JL}$ en el ámbito laboral y su conciliación con la familia.

A un nivel individual, centrándonos en la categoría referente a las exigencias psicológicas, sería interesante poder establecer medidas cuyo objetivo sea facilitar que la cantidad de trabajo sea la más apropiada para el tiempo disponible. Para lograr dicho objetivo, se podrían proponer distintos métodos para mejorar la organización, un reparto de tareas mejor distribuido, estableciendo tareas bien definidas, aumentar las habilidades de liderazgo y potenciar la toma de decisiones en grupo.

En cuanto a las exigencias emocionales, se proponen estrategias de regulación, como técnicas cognitivas que ayuden a no atribuirse un exceso de responsabilidad sobre los resultados de sus alumnos y le permitan desahogarse de manera adecuada. También se puede trabajar el entrenamiento en técnicas de relajación y meditación, para disminuir el nivel de activación fisiológica, aumentar la concentración y mejorar el bienestar subjetivo. Como técnica de relajación la versión reducida de Berstein y Borkovec, ya que su aplicación es más fácil y conlleva menos tiempo que la versión extendida de Jacobson. Por otra parte, se pueden incorporar hábitos de vida saludables como puede ser la realización de ejercicio físico diario, ya que esto puede contribuir positivamente a disminuir su estrés al sentirse más aliviado tanto a nivel físico como psicológico.

Por otro lado, J.L. puede hacer uso de estrategias afectivas como pueden ser autoinstrucciones positivas y autorreforzamiento, de esta manera puede incrementar su autocontrol tanto a nivel laboral como familiar.

En relación a la escala de doble presencia, las medidas a seleccionar deben estar focalizadas principalmente en la facilitación de la compatibilidad de la vida laboral y familiar. Dado que J.L. tiene una familia numerosa, la coordinación y realización de tareas a veces supone ciertas exigencias que pueden influir en la esfera laboral. Con el objetivo de reducir el impacto de este factor psicosocial sobre la salud, se propone realizar un entrenamiento en habilidades de afrontamiento, manejo y distribución del tiempo. Para ello, puede hacer uso de estrategias metacognitivas las cuales incluyen planificación (detallar los pasos a seguir para alcanzar un objetivo propuesto), organización (proponer el momento de inicio y de finalización de consecución del objetivo), y autoevaluación del desempeño (observar cómo se siente de satisfecho con respecto al logro de su meta). 
Aunque el papel individual es fundamental, son muy importantes las medidas que se puedan tomar desde un punto de vista institucional. A continuación, se van a mostrar las más importantes.

A nivel institucional, se pueden tomar medidas para poder lograr reducir el impacto de ciertos aspectos laborales sobre la salud de sus trabajadores. Tomando referencias de grandes empresas como Nestlé, sería necesario racionalizar los horarios, de manera que sean flexibles pero estén limitados para que la jornada laboral no exceda las horas laborales preestablecidas, permitiendo la autogestión de horarios, organización de turnos, posibilidad a las madres y padres de tener turnos especiales por maternidad, reducciones de jornada adaptadas a cada persona, etc. También es importante que exista flexibilidad en el tiempo, esto implica la posibilidad de vacaciones flexibles y días de permisos cuando sean necesarios. Podría ser interesante aumentar la inversión en recursos personales, materiales y servicios para mejorar la conciliación familiar y laboral.

\section{Conclusiones}

A modo de conclusión, se resalta la importancia de evaluación psicosocial de los trabajadores, ya que en el trabajo no sólo interfieren riesgos laborales. De hecho, los riesgos psicosociales pueden llegar a influir en mayor medida que los anteriores, esto se explica haciendo alusión a que la salud del trabajador influye en su rendimiento individual e institucional. De todo esto, se deriva la necesidad de la prevención, evaluación y tratamiento de la salud laboral de los trabajadores e incluso conseguir la promoción de organizaciones saludables.

Además, se apunta que cada uno de estos factores psicosociales objeto de tratamiento deben ser entendidos como dimensiones que en ciertos aspectos se pueden solapar, por lo tanto, el proceso de intervención debe estar orientado a nivel global, pero teniendo en cuenta las individualidades de cada trabajador, es decir, cada una de las técnicas o estrategias usadas en el contexto laboral serán adaptadas a cada profesional según sus necesidades individuales.

\section{Referencias}

Amutio, A. (2004). Afrontamiento del estrés en las organizaciones: un programa de manejo a nivel individual/grupal. Revista de psicología del trabajo y de las organizaciones, 20 (1), 77-94.

Berstein, D.A. y Borkovec, T.D. (1983). Entrenamiento en relajación progresiva: Un manual para terapeutas. $\left(2^{\mathrm{a}}\right.$ edición). Bilbao: Desclée de Brower. (Trabajo original publicado en 1973).

Giner, C.A. (2012). Evaluación de Riesgos Psicosociales en el trabajo. Anales de Derecho, 30, 254-296.

Instituto nacional de seguridad e higiene en el trabajo. (s.f.) Intervención psicosocial en prevención de riesgos
laborales: principios comunes

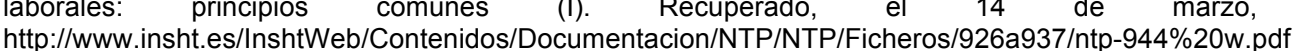

La conciliación de vida familiar y laboral. (s.f.). Recuperado, 14 de marzo de 2015 , de http://www.empresa.nestle.es/es/cvc/equipo-humano/conciliacion-vida-laboral-personal

López-Araujo, B., Osca, A., y Peiró,J.M (2007). El papel modulador de la implicación con el trabajo en la relación entre el estrés y la satisfacción laboral. Psicothema, 19 (1), 81-87.

Moncada, S., Llorens, C., Navarro, A. y Kristens, T.S. (2005). ISTAS21: Versión en lengua castellana del cuestionario psicosocial de Copenhague (COPSOQ). Archivos de Prevención de Riesgos Laborales, 8, 18-29.

Moreno-Jiménez, B., y Garrosa, E. (2013). Salud laboral. Madrid: Pirámide. 
Serrano, M.A., Moya, L., y Salvador, A. (2009). Estrés laboral y salud: Indicadores vasculares y endocrinos. Anales de psicología, 25 (1), 150-159.

Vladut, C.I., y Kalláy, É. (2010). Work stress, personal life and burnout. Cognition Brain and behaviour, 14 (3), 261-280. 